\title{
Fast Phase Aberration Correction In Ultrasound Imaging Using Fat Layer Model
}

\author{
Banazier A. Abrahim, Zeinab A. Mustafa and Yasser M. Kadah \\ Biomedical Engineering Department, Cairo University, Giza, Egypt.
}

\begin{abstract}
Phase aberration is one of the most important factors that limit improvement to lateral resolution of ultrasound imaging system. In this paper we propose a computationally efficient method to correct the phase aberration problem arises from the subcutaneous fat layer. The method is based on the determination of thickness of the fat layer and the velocity in it to calculate the focusing delay perfectly. The thickness and velocity can be determined manually by the user through a qualitative assessment or automatically using a quantitative measure as an objective function. Minimizing the value of the entropy was selected as the cost function. The effect of the fat layer thickness and velocity were simulated as a time delays added to the radio frequency (RF) data. Experimental studies addressing that the entropy can be used to accurately determine the thickness and velocity of the fat layer depending on the selected region of interest (ROI). Images of a six pins phantom were reconstructed by two method in frequency domain by Fourier transform and in time domain, and different images were reconstructed using different aperture size . We drive the evaluation of results simulation in image reconstructed by two method and evaluation phase aberration correction when we used best thickness only and when we used optimum velocity also we do it by execution time and accuracy of image using entropy cost function.
\end{abstract}

Index Terms- Ultrasound imaging, phase aberration ,Image reconstructed, Fourier transform.

\section{INTRODUCTION}

In precent years ultrasound has become one of the most widely used medical modalities because it is safe, noninvasive, portable, easy to use, relatively inexpensive and displays images in real-time[1].

The ultrasound pulse-echo technique have been widely used in medical applications. This techniques using phased array transducers for obtaining a polar cross-sectional image use an array of transducer elements to transmit a focused beam into the body, and each element then becomes a receiver to collect the echoes. The received echoes from each element are dynamically focused to form an image. These systems assume a constant acoustic velocity in the tissue of $1540 \mathrm{~m} / \mathrm{s}$ while steering and focusing the beam. However, soft tissues have a range of acoustic velocities that vary from $1470 \mathrm{~m} / \mathrm{s}$ for fat to $1665 \mathrm{~m} / \mathrm{s}$ for collagen [2]. The acoustic wavefront propagation through a region with locally different acoustic velocities will be phase shifted relative to the rest of the wavefront. As a result, both the received and transmitted pulses are improperly focused and the image quality is degraded. This effect is known as phase aberration of ultrasound images. The effects of phase aberration include a broadening of the point spread function, leading to lower resolution, another effect is increasing the off-axis response, leading to multiple images for the target [3].

The a berator can be modeled as a near field thin phase screen, consistent with the assumption that the layer of subcutaneous fat immediately at the face of the transducer causes the greatest degradation of the beamforming stage [4]. Different techniques have been proposed to correct the phase aberration problem. These techniques are based on measuring and correcting the phase error whether in the time domain or in the frequency domain [5].Ultrasound is used in a wide range of application like land mine detection[6] and personal identification systems[7], the best application of ultrasound is still medical ultrasound imaging[8].

This paper presents a computationally - effective method to correct the phase aberration problem arises from the subcutaneous fat layer. The proposed technique is based on another approach. It does not depend on measuring the arrival time differences but it searches for the best thickness and velocity of the fat layer which achieves the maximum image quality. This algorithm assumes that the acoustic wave is passing through a uniform distributed fat layer with an acoustic velocity is different. Then by determining the thickness of that fat layer, and the optimum acoustic velocity, the steering and focusing delays can be calculated accurately, by using the velocity of the body tissue for the other layer. By this assumption the distortion arises from using the same velocity for all layers can be eliminated by compensating the focusing and steering delays at each element. The images of a six-pins phantom were reconstructed using two method in frequency domain by Fourier transform and in time domain using fat layer model.

We drive the evaluation of results simulation the image reconstructed, we do it by execution time and accuracy of image using entropy cost function, and we evaluation two methods of phase aberration previous and proposed technique.

\section{PREVIOUS WORK}

The area of phase aberration correction has received a wide range of research given its practical importance. Various methods have been described to correct for phase aberration 
in imaging system . Basically two main categories of solution approaches have been proposed in this area. The first is based on cross-correlation between signals from consecutive elements[9],[10], while the other is based on using the speckle brightness as a quality factor[11]. Variants of these methods to improve the robustness of the correction as well as to speed up the calculations were proposed by several authors[1],[12]. We note .However, that the best available computational complexity is still demanding and require fairly expensive hardware components to accomplish its minimum requirements for practicality Hence, a technique that would improve on the accuracy and at the same time provides a simple computationally inexpensive solution would have a large potential in this important area of ultrasound technology.

\section{METHODS}

\section{A. Reconstruction the image(Time domain and Frequency domain)}

After the preparation phase for the raw ultrasonic RF signals, the signal become proper to be used in the image reconstruction process. There were two important preparation steps that must be carried out to the signals before using them. The first step was concerned with increasing the sampling rate for the signals to decrease the quantization error, which may affect the alignment process in the beamformer applied in receive. The second process was to get the signal envelop through Hilbert transform to increase the signal strength, and enhance the reconstruction process.

In this work, Due to the small field- of -view(FOV) of the linear reconstruction and its limited lateral resolution, it was important to use another technique of reconstruction, which applies a steering criterion to increase the FOV. So, the linear phased reconstruction was used to reconstruct the image of the six pins phantom from the data set.

\section{B. Modeling the fat layer}

As mentioned before, the problem of phase aberration arises mainly from the false assumption that assumes constant sound velocity over the FOV So the focusing and the steering delays are calculated based on wrong velocities, which introducing a timing error or phase error. The subcutaneous fat layer participates in this problem, by which the sound velocity propagates in this layer with different velocity[2].

In this paper, we propose a simple method that based on modeling the subcutaneous fat layer as a uniform layer at the face of the transducer with a specified thickness $F_{L}$ such as in Fig. 1. Then by determining the thickness and velocity of this layer the delays can be determined accurately. The time delay was divided into two components; the first component is due to the propagation of the ultrasonic waves in the fat layer, so the delay is calculated based on the path length $L_{f}$ and the velocity of the ultrasonic wave $c_{f}$ in the fat layer is different. The second delay component is due to the propagation of the ultrasonic wave through the entire tissue, which is assumed to have the same velocity $c$, and the delay

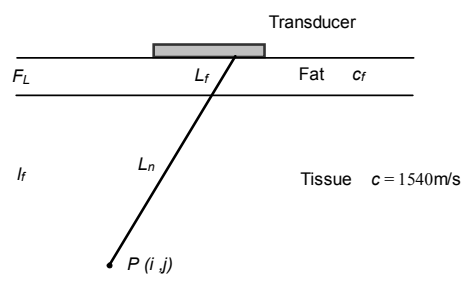

Figure1.Ultrasonic beam from the point target $P$ at depth of focus $I_{f}$ to an aperture element through the tissue layer and the fat layer of thickness $F_{L}$ and velocity $c_{f}$

is calculated based on the path length $L_{n}$ and the velocity in this tissue, which many systems assumed it to be $1540 \mathrm{~m} / \mathrm{sec}$. The total delay $T_{t}$ is calculated using the equation below:

$$
T_{t}=\frac{L_{f}}{c_{f}}+\frac{L_{n}}{c} .
$$

The lengths of $L_{f}$ and $L_{n}$ can be determined from the geometry as shown in Fig. 1, and the length $L_{f}$ can be determined as follow:

$$
L_{f}=\frac{F_{L} * R_{n}(i, j)}{l_{f}} .
$$

Here $R_{n}(i, j)$ is the distance from the center of the element to the point target $P(i, j)$.

\section{Fat Layer Thickness and Velocity}

There are two proposed methods to determine thickness and velocity of the subcutaneous fat layer. The first is a qualitative method depends on the user judgment, and the second is quantitative method using a simple automatic algorithm.

In first method the user can change the thickness of the fat layer manually from a thickness of $0 \mathrm{~mm}$ to $55 \mathrm{~mm}$ and velocity $1450 \mathrm{~m} / \mathrm{s}$ to $1640 \mathrm{~m} / \mathrm{s}$ via a specific knob in the device keyboard like the knob which change the depth of focus, or the power density. The resolution of the increment can be set to be $5 \mathrm{~mm}$ in thickness and $10 \mathrm{~m} / \mathrm{s}$ in velocity. By using this knob, the user can determine qualitatively the thickness of the fat layer that achieves the maximum image quality during the device use.

The second method depends on a simple algorithm that can automatically determine the thickness and velocity of the fat layer. This algorithm reconstructs the image, or a ROI with in the image, calculates the point spread function (PSF) for the imaging system at different fat layer thicknesses and velocity. Then it searches for the thickness and velocity which achieves the minimum value for a specific cost function. This value achieves the highest image quality, and the minimum bad effects such as image blurring. When the true thickness and velocity are determined, the whole image can be reconstructed. In this paper the value of entropy is used as the cost function.

When the value of the entropy decreases the energy distribution within a region of interest in the image becomes 
of higher contrast and has sharper edges, i.e. as the effect of image blurring decreases as the value of the entropy decreases within a specified ROI within the image. Moreover, it was observed that as PSF of the imaging system becomes more precise (of higher main loop and small side lobes) as the value of the entropy becomes lower. The algorithm selects a specific ROI within the image and calculates its value of entropy at different assumed thicknesses and velocity for the fat layer. The best thickness and velocity for the fat layer will be at the minimum value of entropy. The type of entropy used in this algorithm was the (nonnormalized) Shannon entropy, which can be calculated using the following equation:

$$
E(s)=-\sum_{i} s_{i}^{2} \log \left(s_{i}^{2}\right) .
$$

Here $s$ is the signal and $\left(s_{i}\right)_{\mathrm{i}}$ the coefficients of $s$ in an orthonormal basis[13].

\section{Fourier based phase aberration estimation and image reconstruction}

From the Fourier shift theorem, the image reconstructed and the time delay computed from the cross-correlation based methods corresponds to the group delay from the phase difference between the two signals of interest in the Fourier domain. This may imply that we can work within this parallel universe to compute the group delay and converge as the cross-correlation technique to the time delay corresponding to the present phase aberration. However, this is not practical for the following reasons:

a. It is not straightforward to compute the group delay from the phase difference between the two signals as a result of the presence of severe phase wrapping. The problem of phase unwrapping is a challenging one in practice and many techniques are not robust enough for our problem.

b. The computation of the Fourier transform for the all signals is computationally costly to start with given that we work with the significantly larger set of RF samples[5].

Here, we propose a simple, computationally efficient and non-iterative method to obtain a fairly accurate estimate for the time delay corresponding to phase aberration based on the Fourier transform method. The basic idea behind this technique is to align the signal components from all elements only at the center frequency of the probe. The proposed technique works as follows:

a. Compute the center frequency of the probe as the maximum of the Fourier transform of only one of the signals.

b. Compute only the frequency component corresponding to the center frequency for all elements using the basic discrete Fourier transform (DFT) formula.

c. Compute the phase differences between the computed center frequency components related to a chosen reference. signal in the aperture. Here, we used a central element as our reference.

d. Estimate the relative delays as the phase differences divided by the center frequency. e. Construct the phase conjugate filter.

f. Apply the phase conjugate filter to the received signal, to remove the decorrelation in phase spectra caused by aberration.

\section{E. Evaluation of several methods (accuracy and time) and computer simulation}

In this paper we evaluation the results simulation in image reconstructed and phase aberration correction when determine the best thickness only and when best velocity is determine also, by two method, we do it by execution time and accuracy of image using entropy cost function.

\section{RESULTS AND DISCUSSION}

The proposed methods were applied to correct real data obtained from the Biomedical Ultrasound Laboratory, University of Michigan. Although the techniques proposed were applied to several data sets, the data set that was used to generate the results in this paper is the one under "Acuson 17".

The parameters for this data set are as follows: 128 channels, 13.8889 MSPS A/D sampling rate, 3.5 MHz transducer with $0.22 \mathrm{~mm}$ element spacing, $2048 \mathrm{RF}$ samples per line each represented in 2 bytes, and 8 averages.

The data were acquired for a phantom with six pins at different positions.

We used the data to simulate the N-channel beamformer on receive. The radio frequency (RF) signals, A-scans, were recorded from every possible combination of transmitter and receiver for all elements in the 128 element.

In Figs. 2 and 3, show an illustration of the magnitude and phase Fourier transform of the signals obtained from the 48 aperture elements respectively.

The horizontal direction represents the frequency while the vertical corresponds to the element position in the aperture.

Note that the magnitude information appears invariant with element position while the phase information shows quite a bit of variability, because of this the a berrator can be modelled as a near field thin phase screen and the image reconstructed from phase of signal.

In Fig. 4, illustration the estimated variations of phase between different samples this phase used to calculate delays using the Fourier based method.

In Fig. 5, the result of typical RF signal for phantom show the six peaks of phantom pins is illustrated.

Images of a one pin phantom were reconstructed by two method in frequency domain by Fourier transform and in time domain, and different images were reconstructed using different aperture size. This pin is shown it in Figs. 6 and 7.

We observe the time execution in frequency domain is very short than time domain and low computation complexity, simplicity make this method very fast, but time domain method shows better lateral resolution than the frequency domain the pin is shown in frequency domain is not optimum. 


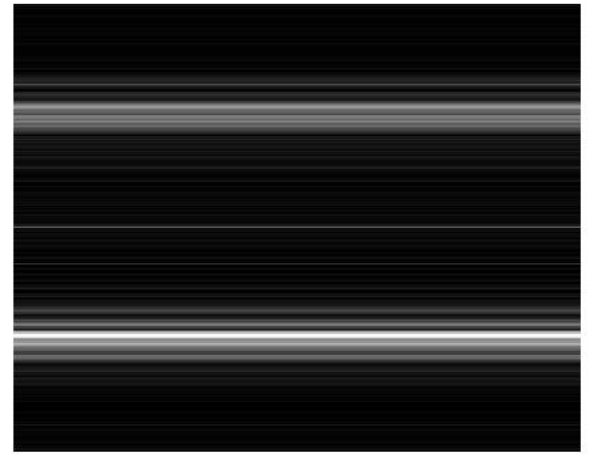

Figure 2. Illustration of the magnitude of the Fourier transform of a single element data. The horizontal direction represents frequency while the vertical

is element number. Note the similarity between different elements.

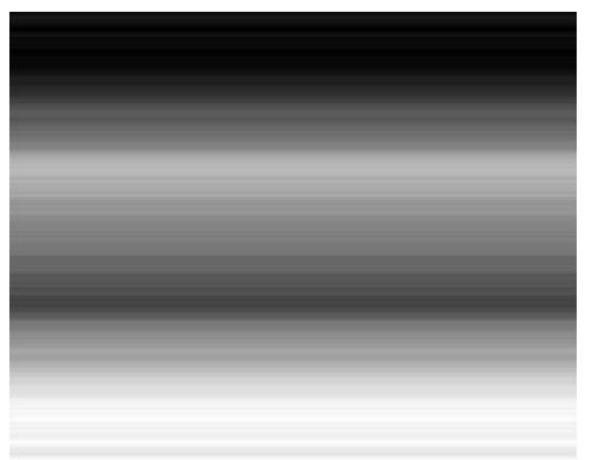

Figure 3. Illustration of the phase of the Fourier transform of a single element data. The horizontal direction represents frequency while the vertical is element number. Note the variations between different elements within the areas with significant magnitude.

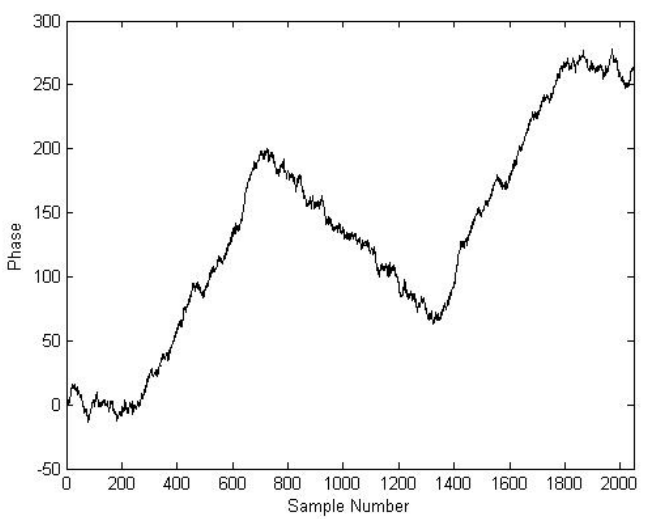

Figure 4. Illustration the estimated variations of phase between different samples using the Fourier based method.

The result in time domain we do it by using fat layer model. The result of the linear reconstruction technique indicate that the lateral resolution of the images, is improved by increasing the aperture size during reception. It is observed that linear reconstruction is not efficient to cover a wider FOV, which is important for general ultrasound application. So, another reconstruction technique was applied, and the main concern

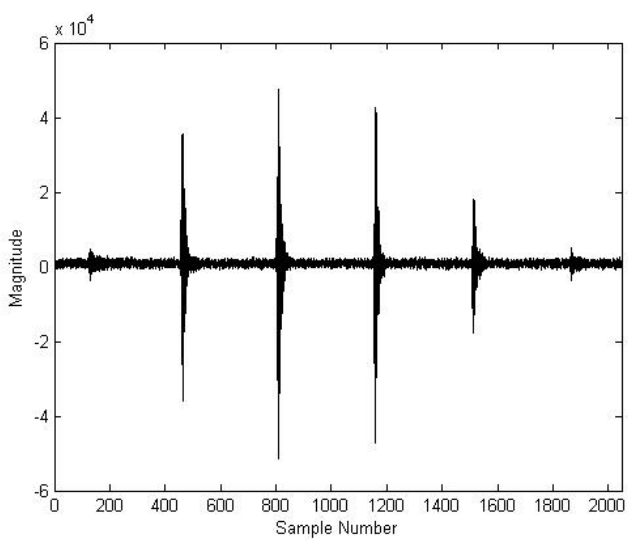

Figure 5. Illustration typical RF signal for phantom shows the six peaks of phantom pins.

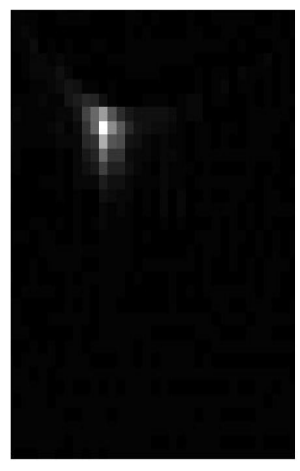

(a)

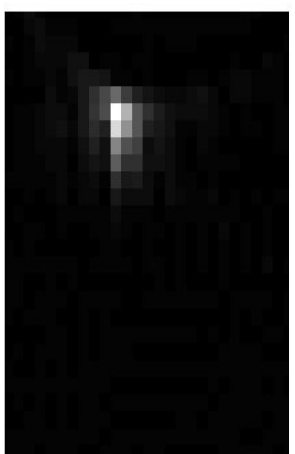

(b)
Figure 6. Illustration Ultrasound image reconstructed for one pin of the phantom in time domain(a) before phase correction(b)after phase correction.

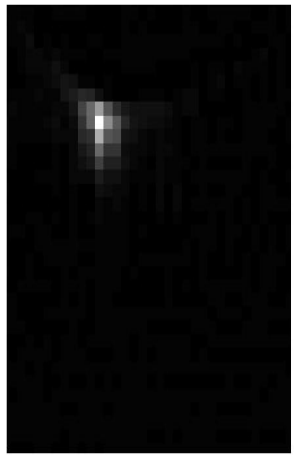

(a)

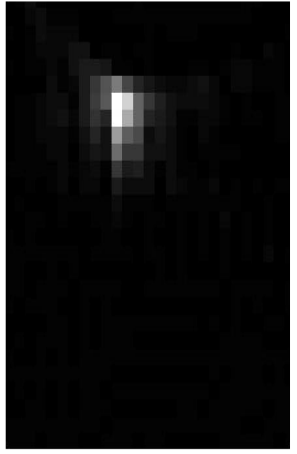

(b)
Figure 7. Illustrates Ultrasound image reconstructed for one pin of the phantom in frequency domain (a) before phase correction(b)after phase correction.

in the technique is to reconstruct the image of the total FOV, all the six pins of the phantom, for example, with a sufficient lateral resolution. The second technique depends on both steer and focus the ultrasound beam to cover the whole FOV. The digital beamforming algorithm described in the second technique is very simple depending on the raster points rather 
than the radial focal points. So, there is no need for the scan conversion from the radial format to the raster format, which will decrease the mathematical complexity of interpolation and the reconstruction time. The beamforming was used to reconstruct the six pin image of the phantom and other images from different datasets. The second reconstruction technique is used as a primary procedure in the process of phase aberration correction.

The effect of the subcutaneous fat layer was simulated during the image reconstruction stage of the data set (Acuson17) for the six pins phantom. The data set was used to reconstruct the first point of the phantom and shows the degradation of the image quality at different thicknesses of the fat layer. When the image reconstructed without aberration effect ( $0 \mathrm{~mm}$ fat layer), the point appears precise and focused. As the aberration effect is introduced during the image reconstruction phase, the spatial resolution is degraded in both the axial and lateral directions, when the image was reconstructed at fat layer thicknesses $(5 \mathrm{~mm}$ to $55 \mathrm{~mm})$. Obviously, you can be observed that the degradation of the spatial resolution increases as the thickness of the fat layer increase but when obtain the optimum velocity in fat layer the result may be change this is propose of paper.

The aberration effect can be studied by analyzing the system PSF at different fat layer thicknesses. The degradation of the PSFs is clearly explained, which includes the broadening of the PSF, the decreasing of the magnitude of the main lobe, and the increasing of the magnitude of the side lobes[14]. Fig. 8, represents the PSF of the system with zero fat layer (no aberration), and it shows a high magnitude and sharp main lobe and small side lobes. The degradation effect increases with the thickness of the fat layer increase at $70 \mathrm{~mm}$ fat layer the main lobe decreases, and becomes more broadened, and the magnitude of the side lobes increases.

It is observed that the minimum value of the entropy exists perfectly at $0 \mathrm{~mm}$ fat layer thickness compare to $70 \mathrm{~mm}$ fat layer thickness at normal velocity on fat layer $1470 \mathrm{~m} / \mathrm{s}$.

In Fig. 9, it is observed that when the thickness change ( $5 \mathrm{~mm}$ to $55 \mathrm{~mm})$ the minimum value of the entropy exists perfectly at the $15 \mathrm{~mm}$ simulated thicknesses, when we use normal velocity $1470 \mathrm{~m} / \mathrm{s}$, the entropy values are calculated for the system PSF at thickness.

In Fig. 10, the proposed technique use optimum velocity and best thickness together, the thickness $50 \mathrm{~mm}$ at $1560 \mathrm{~m} / \mathrm{s}$ become better and the value of entropy become minimum than previous technique when we use thickness $15 \mathrm{~mm}$ at $1470 \mathrm{~m} / \mathrm{s}$, the effect in PSF also illustration it in Fig. 11.

The phase correction method explained previously is very simple. It depends mainly on the determination of the thickness and velocity of the subcutaneous fat layer to estimate the focusing delay correctly. The entropy was used as the cost function to estimate the thickness and velocity of the fat layer introduces perfect results as shown in Figs. 10 and 11, at different fat layer thicknesses and velocity with an accepted error of $\pm 1 \mathrm{~mm}$, but these results were obtained when a specific ROI within the image was used to calculate the entropy. The accuracy may differ totally when another ROI is used. The accuracy was improved when the entropy of different ROIs were added, therefore the error can be reduced.

The entropy of the PSF at different thicknesses and velocity can be used in the algorithm and then searching for the minimum entropy value is required. At this minimum value the optimum thickness and velocity exist. So, in the automatic algorithm for determining the thickness and velocity of the fat layer, there is no need to reconstruct the whole image. The values of the entropy of the PSFs can be calculated or a partial area of the image can be reconstructed using the simple beamforming algorithm, raster point algorithm.

The value of the velocity in fat layer is important factor to estimate the focusing delays.

After the estimation of the fat layer thickness and velocity, the corrected image can be totally reconstructed by the true focusing delays. The simplicity and low computational complexity (algorithm complexity) make this solution very

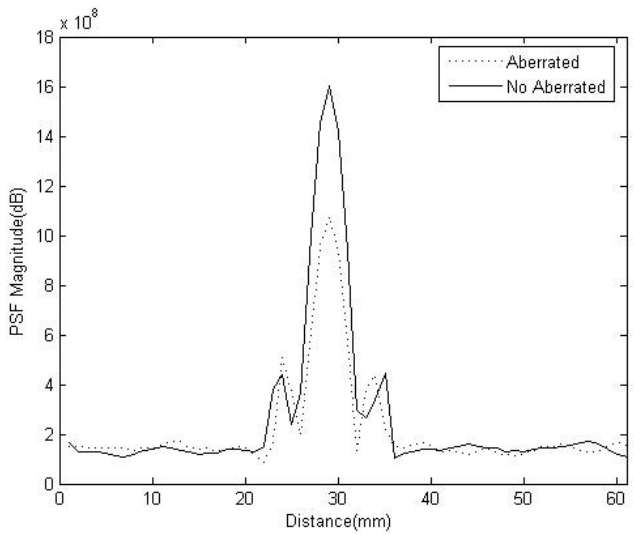

Figure 8. Tow dimensional point spread function at $0 \mathrm{~mm}$ fat layer thickness(No Aberrated), 70mm fat layer thickness(Aberrated).

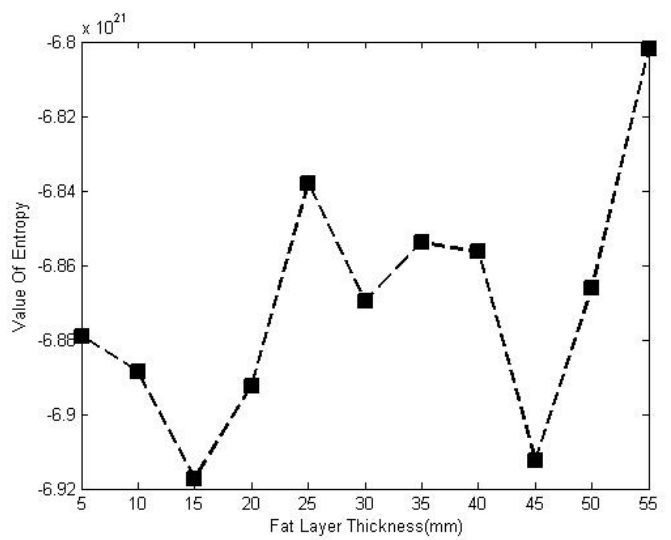

Figure 9. The change of the value of entropy with assumed thickness of the fat layer: $(5 \mathrm{~mm}$ to $55 \mathrm{~mm})$ at normal velocity $1470 \mathrm{~m} / \mathrm{s}$, the minimum value of the entropy exists at $15 \mathrm{~mm}$. 


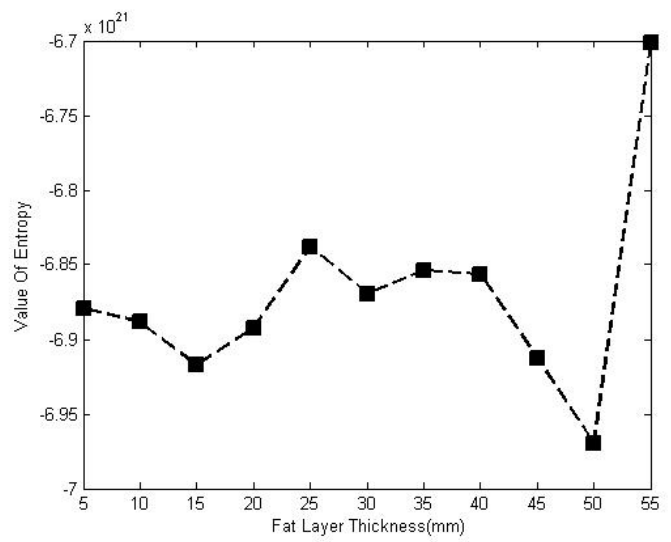

Figure 10. The change of the value of entropy with assumed thickness of the fat layer: $(5 \mathrm{~mm}$ to $55 \mathrm{~mm})$ at optimum velocity $1560 \mathrm{~m} / \mathrm{s}$, the minimum value of the entropy exists at $50 \mathrm{~mm}$.

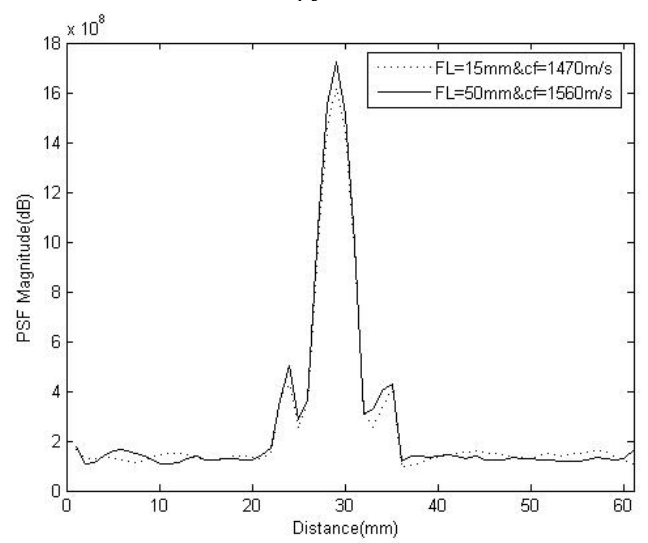

Figure 11. Tow dimensional point spread function at $15 \mathrm{~mm}$ fat layer thickness at $1470 \mathrm{~m} / \mathrm{s}, 50 \mathrm{~mm}$ fat layer thickness at $1560 \mathrm{~m} / \mathrm{s}$.

fast, whereby the computational complexity is linear rather than quadratic for previous methods based on correlation. The main disadvantage is that the accuracy is dependent on the selected ROI. Future work should therefore focus on this problem and experimental studies will be performed. It is important to mention that, the described correction scheme is the first solution that depends on in vivo measurements for the fat layer thickness.

\section{CONCLUSIONS}

We propose a computationally-effective method for the problem of phase aberration in ultrasound images resulting from the subcutaneous fat layer. The new method includes a planar model for fat layer is assumed and relies on calculating the time delay taking into account the difference in the ultrasonic velocity between the fat and the tissue. The determination of the thickness and velocity of the fat layer can be estimated by two methods, and this method is better than determined the thickness only. The first is manually by user through a qualitative assessment and the second method is automated based on Shannon entropy as a cost function. Experimental results from both simulations and real data acquired from two different ultrasound systems from a beamforming algorithm applied on receiving mode were used to verify the solution using images reconstructed based on raster points in frequency and time domain. The results indicate that a significant improvement using the proposed methods and suggest their practicality and clinical utility, and the important of time domain to allows more precise implementation of delay and image reconstruction . Further work is needed to use another searching technique to look for the minimum value at a short time to estimate the best thickness and velocity to estimate the focusing delay correctly and the problem of algorithm dependency on the ROI must be studied in the future to improve the accuracy of this method.

\section{ACKNOWLEDGMENTS}

The authors would like to thank Dr. Matthew O'Donnell for making their data available to us. Also, This work was supported also in part by the IBE Tech Giza, Egypt.

\section{REFERENCES}

[1] P.D. Freiburger and G.E. Trahey, "Parallel processing techniques for the speckle brightness phase aberration correction algorithm," IEEE Trans. Ultrason., Ferroelect., and Freq. Contr. 44, no. 2, pp. 431-444, 1997.

[2] S.A. Gross, R.L. Johnston, and F. Dunn, "Comprehensive compilation of empirical ultrasonic properties of mammalian tissues, "J. Acoustic. Soc. Amer., vol. 64, pp. 423-457, 1978.

[3] G.C. Ng, S.S. Worrell, P. D. Freiburgger, and G. E. Trahey, "A comparative evaluation of several phase aberration correction algorithms," IEEE Trans. Ultrason., Ferroelec. Freq. Contr. , vol. 41, no. 5 , pp. 631-643, 1994.

[4] G. C. Ng. P. D. Freiburgger, W. F. Walker, and G. E. Trahey, " A speckle Target Adaptive Imaging Technique in the presence of distributed aberration," IEEE Trans. Ultrason., Ferroelec. Freq. Contr. vol. 44, no. 1, pp. 140-151, 1997.

[5] Z. Farouk, A. M. Youssef, and Y. M. Kadah, "Successive optimization for fast aberration correction," Proc. SPIE Medical Imaging 2003, San Diego , 2003.

[6] D. Donskoy, A. Ekimon, N. Sedunov, and M. Tsionsky, "Nonlinear seismo acoustic land mine detection and discrimination, "J. Acoust. Soc. Am., vol. III,no.6,pp.2705-2714,2002.

[7] J. K. Schneider, and S. M. Gojevic, " Ultrasonic imaging for personal identification," in proc.2001 IEEE-UFFC Ultrasonics Symposium, pp.595-601,2001.

[8] S. L. Hagen- Ansert, Textbook of Diagnostic Ultrasonography, $5^{\text {th }}$ ed.St.Louis: C.V.Mosby, 2000.

[9] M. O'Donnell and S.W. Flax, "Phase-aberration correction using signals from point reflectors and diffuse scatterers: measurements," IEEE Trans. Ultrason., Ferroelect., and Freq. Contr. 35, no. 6, pp. 768-774, 1988

[10] S. W Flax and M. O'Donnell," Phase aberration correction using signals from point reflectors and diffuse scatterers: basic principles," IEEE Trans. Ultrason., Ferroelec. Freq. Contr. , vol. 35, no. 6, pp. 758-767, 1988.

[11] L. F. Nock, G. E. Trahey, and S.W. Smith, " Phase aberration correction in medical ultrasound using speckle brightness as a quality factor," J. Acoust. Soc. Am., vol. 85, no. 5, pp. 1819-1833, 1989.

[12] M. Karaman, A. Atalar, H. Koymen, M. O'Donnell, "A phase aberration correction method for ultrasound imaging," IEEE Trans. Ultrason., Ferroelec. Freq. Contr. , vol. 40, no. 4, pp. 275-282, 1993.

[13] R.R. Coifman, M.V. Wickerhauser, "Entropy-based Algorithms for best basis selection," IEEE Trans. on Inf. Theory, vol. 38, no. 2, pp. 713-718, 1992.

[14] A. M. Ehab, Z. Farouk, A. M. Youssef, and Y.M. Kadah, "Ultrafast Phase Aberration Correction in Ultrasound Imaging Using a Simple Model for fat layer," in Proceeding $23^{\text {rd }}$ National Radio Science Conference, Menoufiya University, Egypt, vol. k, no. k1, pp. 1-8, 2006. 